\title{
Bilateral scaphoid stress fracture in a platform diver presenting with unilateral symptoms
}

Nor Hazla Mohamed Haflah ${ }^{1}$, MBChB, MS, Noreen Fazlina Mat Nor ${ }^{1}$, MD, Shalimar $\underline{A b d u l l a h}^{1}$, MBBS, MS, Jamari Sapuan ${ }^{1}$, MD, MS

\begin{abstract}
Scaphoid stress fracture is rare and occurs mainly in gymnasts. The current literature has only two reported cases: unilateral scaphoid stress fracture in a platform diver and bilateral scaphoid stress fracture in a gymnast. We herein report bilateral stress fracture of the scaphoid in a platform diver who presented with only one symptomatic side. Our patient was a 16-year-old competitive platform diver with an 18-month history of pain in the right wrist. Radiography revealed fracture of the right scaphoid at the waist. As part of our preoperative plan of measuring the scaphoid length to determine the appropriate screw, radiography of the contralateral side was performed, revealing an unexpected fracture of the left scaphoid. Due to the frequency of stress fractures in competitive sports, especially gymnastics, we recommend that bilateral scaphoid radiography be performed for athletes presenting with a unilateral scaphoid fracture, to avoid missing a fracture in the contralateral side.
\end{abstract}

Keywords: bilateral, platform diver, scaphoid, stress fracture

\section{INTRODUCTION}

Diving is commonly associated with spinal cord injuries.(1) Scaphoid stress fracture is rare, occurring mainly in gymnasts..$^{(2-7)}$ There are only two reported cases in the current literature: one case of unilateral scaphoid stress fracture in a platform diver and another of bilateral scaphoid stress fracture in a gymnast. ${ }^{(2,4)}$ We herein present a case of bilateral stress fracture of the scaphoid in a platform diver who presented with only one symptomatic side.

\section{CASE REPORT}

A 16-year-old, right-handed male diver in the national team presented with pain and swelling of the right wrist. He first noticed the pain 18 months prior to his presentation at our centre. The pain, which was initially described as mild, gradually increased in intensity. There was no history of trauma that could account for the symptoms. Consequently, he had to discontinue his training. At a regional health centre, the diagnosis of wrist fracture was made, for which he was advised surgery and referral to an orthopaedic surgeon. However, the patient declined and sought treatment from a traditional healer.

Over the course of one year, the patient's pain worsened. He then consulted a doctor at a district hospital. Radiography of the right wrist showed nonunion of the scaphoid fracture, with sclerotic edges, bone resorption and cystic changes (Fig. 1). Surgery was recommended, which he again declined but agreed to undergo physiotherapy. Since the onset of wrist pain, the patient had completely stopped his training. When his symptoms did not improve with physiotherapy, he finally agreed to undergo surgery and was referred to our centre for surgical intervention.

At our centre, examination of the patient revealed tenderness over the anatomical snuff box of the right wrist. His range of motion (ROM) was decreased, and he was unable to actively

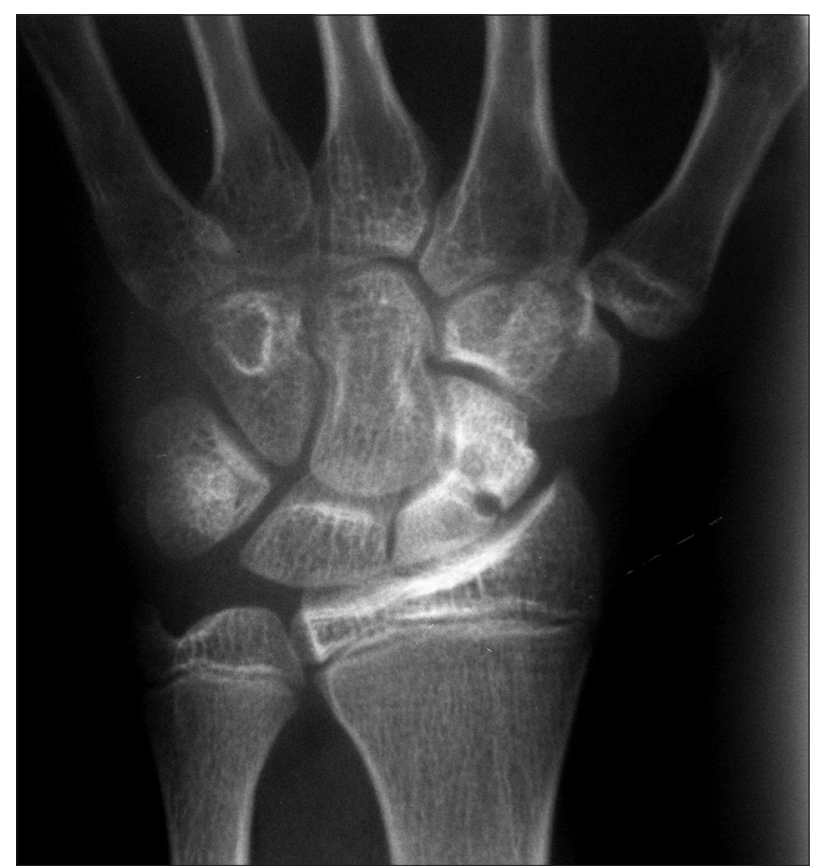

Fig. 1 Radiograph of the right wrist shows scaphoid nonunion with sclerotic fracture edges, bone resorption and cystic changes.

dorsiflex his right wrist. Passive dorsiflexion was $30^{\circ}$, both active and passive palmarflexions were $30^{\circ}$, and pronation and supination were $50^{\circ}$ and $40^{\circ}$, respectively. Grip strength, measured using the Jamar dynamometer, was $18 \mathrm{~kg}$.

As part of our preoperative plan of measuring the scaphoid length to determine the appropriate scaphoid screw, radiography of the contralateral scaphoid was performed, revealing an unexpected stress fracture of the left scaphoid (Fig. 2). On further enquiry, the patient denied any history of pain in the left wrist. On examination, there was no tenderness over the left anatomical 


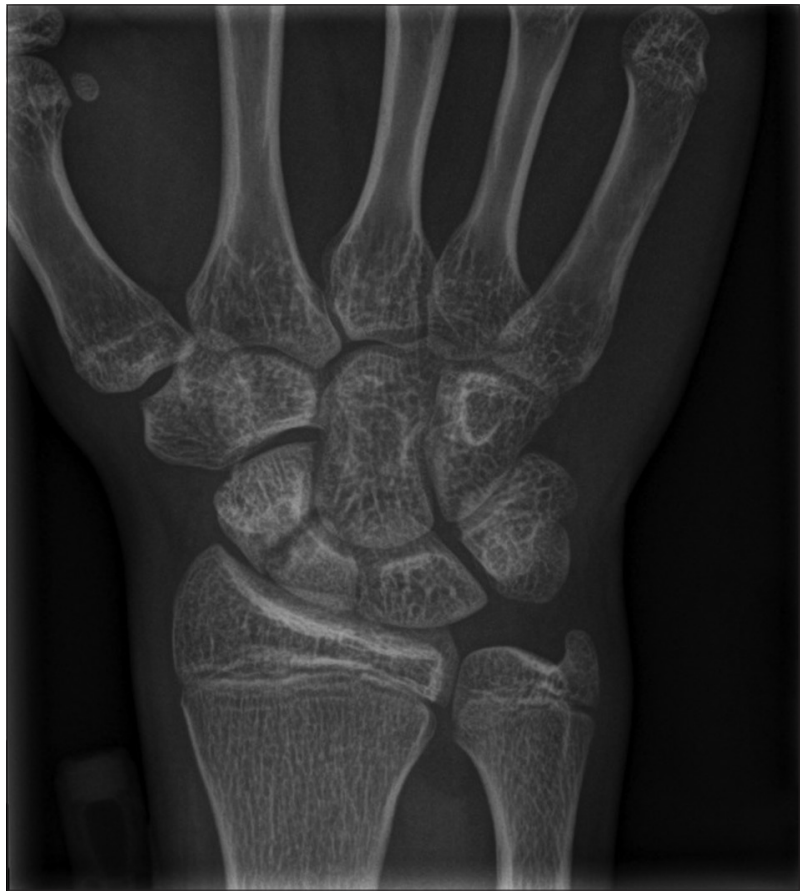

Fig. 2 Radiograph of the left wrist shows stress fracture of the left scaphoid

snuff box or on axial compression. The ROM of his left wrist was full and grip strength was $14 \mathrm{~kg}$, as measured by the Jamar dynamometer. In light of this new finding, we decided to perform screw fixation and bone grafting for both the scaphoid fractures.

Surgical interventions on both scaphoids were done via the standard volar approach, exposing the fracture site between the flexor carpi radialis and radial artery. Both fracture sites were located at the waist of the scaphoid with punctuate bleeding. The Acutrak ${ }^{\circledR}$ Headless Compression Screw (Acumed, Hillsboro, Oregon, USA) was used to fix each fracture, and cortical iliac bone graft was added to promote union.

Intraoperatively, the fixation was stable on all movements of the wrist joint. Postoperatively, a back slab was initially applied prior to conversion to a full scaphoid cast. The patient was symptom-free at four months post operation. The ROM for both wrists was full, and grip strengths on the right and left sides were $22 \mathrm{~kg}$ and $14 \mathrm{~kg}$, respectively. Radiography confirmed fracture union (Fig. 3).

\section{DISCUSSION}

Stress fracture of the scaphoid is rare. ${ }^{(2-5)}$ Most reported cases are of gymnasts who repeatedly exert large amounts of force on their wrists. ${ }^{(4-7)}$ Patients usually complain of longstanding wrist pain that may intensify over time before they eventually seek medical opinion. The absence of trauma may help to differentiate between an acute fracture and a stress fracture. ${ }^{(6)}$

Diving is usually associated with cervical spine injury. ${ }^{(1)}$ Prior to the present case, there has only been one other reported case of scaphoid stress fracture in a diver. The mechanism of stress loading in a diver may occur when he or she hyperextends the wrist when entering the water. Another point of injury is when divers perform handstands on the diving board, akin to that performed by a gymnast. ${ }^{(2)}$ In highly trained athletes, repeated

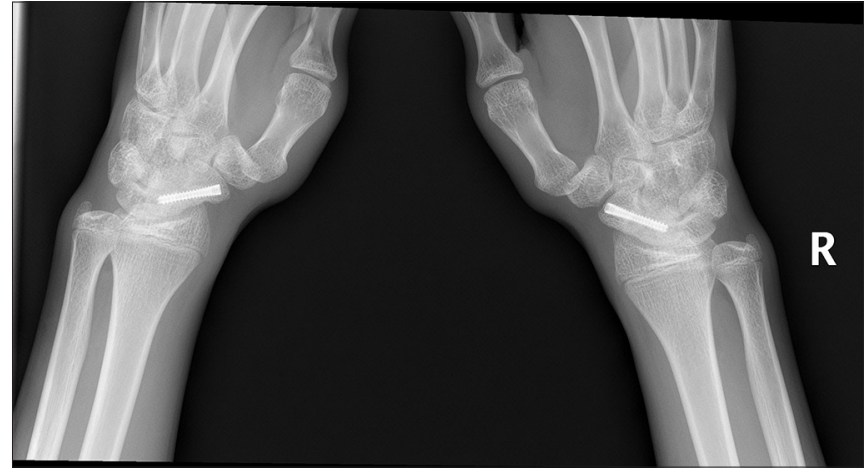

Fig. 3 Radiograph of the bilateral wrists, taken four months after surgery, shows union of both fractures, with screws in situ.

hyperextension and muscle fatigue have been postulated to be causal factors in stress fractures.

Brutus and Chahidi ${ }^{(3)}$ reported an unusual case of scaphoid stress fracture in a badminton player who experienced a sudden onset of pain, in contrast to the gradual onset of pain in stress fracture. Unlike in gymnasts, the mechanism of injury in this case was most likely repeated flexion and extension of the wrist, as loading forces in the wrist are unlikely in badminton.

Our patient had bilateral stress fracture of the scaphoid that was only symptomatic on one side. In view of the presentation, a possible differential diagnosis could be bilateral bipartite scaphoid. However, several factors appear to indicate otherwise. Firstly, Bunnell's criteria require the absence of a history of trauma in order to support a diagnosis of bipartite scaphoids; ${ }^{(8)}$ our patient, however, had a history of repeated loading stress or trauma. Secondly, the radiographic features demonstrated a stress fracture with an interrupted cortical border or a lack of cortical rimming at the opposing edges of the gap. There is also the presence of a cystic lesion around the fracture site, which may appear before the occurrence of stress fractures. ${ }^{(4)}$ Thirdly, our intraoperative finding shows a break in the cortex, consistent with a fracture. Finally, Louis et al refuted the existence of congenital bipartite scaphoids, believing it to be a traumatically acquired condition. ${ }^{(9)}$

Scaphoid stress fractures in athletes can be very difficult to detect clinically, as such fractures do not have a history of trauma. Thus, patients typically present late (delays of six weeks to three months) with radiological features supporting nonunion. ${ }^{(2,5,6)}$ The absence of positive findings in plain radiography, however, does not exclude a stress fracture. Matzkin and Singer reported a case in which signs of fracture were absent on radiography at three months but became obvious at six months. ${ }^{(6)}$ Therefore, any radial or dorsal wrist pain in at-risk athletes should warrant a close examination for signs of scaphoid fracture. If a fracture cannot be detected on plain radiography, computed tomography or magnetic resonance imaging should be considered whenever there is clinical suspicion.

Stress fractures can be treated either surgically or conservatively. Matzkin and Singer, and Hanks et al both reported successful outcomes with conservative treatment for stress fractures in gymnasts. ${ }^{(6,7)}$ In our patient, treatment of the established nonunion and prevention of scaphoid nonunion advanced collapse were the indications for surgery at 18 months after the onset of pain. In contrast to Matzkin and Singer's patient, 
who demonstrated healing at six months post operation, ${ }^{(6)}$ our patient achieved scaphoid union at four months.

In conclusion, due to the frequency of stress fractures in competitive sports, we recommend that bilateral scaphoid radiography be performed for athletes presenting with a unilateral scaphoid fracture, to avoid missing a fracture in the contralateral side.

\section{REFERENCES}

1. Kluger Y, Jarosz D, Paul DB, Townsend RN, Diamond DL. Diving injuries: a preventable catastrophe. J Trauma 1994; 36:349-51.

2. Hosey RG, Hauk JM, Boland MR. Scaphoid stress fracture: An unusual cause of wrist pain in a competitive diver. Orthopedics 2006; 29:503-5.

3. Brutus JP, Chahidi N. Could this unusual scaphoid fracture occurring in a badminton player be a stress fracture? Chir Main 2004; 23:52-4.

4. Engel A, Feldner-Busztin H. Bilateral stress fracture of the scaphoid. A case report. Arch Orthop Trauma Surg 1991; 110:314-5.

5. Nakamato JC, Saito M, Medina G, Schor B. Scaphoid stress fracture in high-level gymnast: A case report. Case Rep Orthop 2011; 2011:492407.

6. Matzkin E, Singer DI. Scaphoid stress fracture in a 13-year-old gymnast: a case report. J Hand Surg Am 2000; 25:710-3.

7. Hanks GA, Kalenak A, Bowman LS, Sebastianelli WJ. Stress fracture of the carpal scaphoid. J Bone Joint Surg 1989; 71:938-41.

8. Boyes JH. Fractures and dislocations. In: Bunnell's Surgery of the Hand. 5th ed. Philadelphia: JB Lippincott, 1970: 592-5.

9. Louis DS, Calhoun TP, Garn SM, Carroll RE, Burdi AR. Congenital bipartite scaphoid--fact or fiction? J Bone Joint Surg Am 1976; 58:1108-12. 